\title{
The "foot-in-the-door" phenomenon: Mediating effects of size of first request and sex of requester*
}

\author{
ROBERT A. BARON \\ Purdue University, West Lafayette, Ind. 47907
}

The effects of prior exposure to an initial request of small or moderate magnitude on later willingness to comply with a much larger request were examined. It was predicted that $S s$ exposed to a moderate initial request would be more likely to comply with the later, large, request than those exposed to only a small initial request, and that individuals in both of these groups would show a higher rate of compliance than those in a control condition who were contacted only once and immediately asked the large request. Contrary to these predictions, however, results indicated that only exposure to the small initial request was successful in increasing later compliance.

In two recent experiments, Freedman \& Fraser (1966) have demonstrated that compliance with relatively large requests from a stranger may be significantly increased by prior exposure to a smaller, initial, request. More specifically, these investigators found that Ss visited or telephoned in their own homes and asked to perform a large request for the $\mathrm{E}$ were much more likely to agree if they had previously been asked to perform a much smaller favor for the same or another individual than if they had not. Freedman and Fraser termed this phenomenon the "foot-in-the-door" effect and suggested that it might provide a useful means of attaining increased compliance with various requests in the absence of overt social pressure.

The present experiment was designed to extend this initial work by investigating the influence of a factor that might reasonably be expected to affect the magnitude of the "foot-in-the-door" phenomenon, the size of the initial small request. In particular, it was designed to investigate the hypothesis that within certain determinable limits, the larger the first request to which Ss are exposed, the greater their subsequent tendency to comply with a second request of more substantial magnitude. This hypothesis was based upon the assumption that the greater the size of the first request, the smaller, in comparison, will the second appear to be (see, e.g., Helson, 1948). Hence, the greater the rate of compliance with this latter request on the part of Ss.

In order to investigate the accuracy of this prediction, Ss in two different experimental groups were approached in their homes on a first occasion and asked to perform either a small or moderate-sized task for the E. One week later they were visited again and asked to perform a much larger task. Individuals in a third (control) condition were approached only once and asked at that time to perform the large request. On. the

*The author wishes to express his appreciation to Anne Cothran, Fred Duchac, Bud Gore, and Carol Moore for their able assistance in the collection of the data. basis of the reasoning described above, it was predicted that Ss exposed to the moderate initial request would show a higher rate of compliance with the second, large, request than Ss exposed to the small initial request. In addition, in accordance with the findings of the Freedman \& Fraser (1966) experiment, it was expected that Ss in both of these groups would show a higher level of compliance with the large request than would Ss in the one-contact control condition.

\section{METHOD \\ Subjects}

Ss were 120 individuals ( 98 females, 22 males) residing in a white, middle-class section of Columbia, S.C. They ranged in age from approximately 18 to approximately 65 . However, most were middle-aged housewives in the age bracket of $35-45$. Ss' age and sex did not vary across experimental conditions.

\section{Two-Contact Groups \\ Procedure}

Ss in the two-contact conditions were visited in their homes on two separate occasions. In the first instance, they were approached by one of the four Es (two males, two females) and asked to perform one of two requests. In the small-first-request condition, they were simply asked to accept a one-page leaflet describing the dangers of air and water pollution, while in the moderate-first-request condition, they were asked to sign a petition calling for stricter antipollution legislation, to get two of their friends to sign copies of the same document, and return all three forms in a stamped, addressed envelope provided by the $\mathrm{E}$. These requests were chosen on the basis of pretests conducted with 70 college students which indicated that they differed significantly in perceived size, but would elicit similar, high rates of compliance.

In both conditions, the Ss introduced themselves as members of "South Carolinians for a Clean Environment" (a fictitious group) and followed a prepared speech in making the appropriate request. In both cases, they attempted to elicit a definite "yes" or "no" from the S, and politely discouraged prolonged conversations. As a precaution against communication between Ss, the four Es worked on different but adjacent streets and visited only every other house on each block. In addition. when beginning a new condition, they moved to a new (but adjacent) block so as to prevent neighbors from falling into different experimental treatments.

One week after the initial contact, the two male and two female Es exchanged address lists and then returned for a second visit. Thus, on this occasion, Ss were approached by an $E$ of the same sex as the one who had visited them before, but by a different specific individual. In this manner, Es were prevented from knowing in advance the experimental condition to which Ss had been assigned on the first visit. As on this previous occasion, they introduced themselves as members of "South Carolinians for a Clean Environment." However, in this instance they made a much larger request than was the case previously, asking the Ss' permission to place a large $(3 \times 5 \mathrm{ft})$ sign on their front lawns. The sign ostensibly contained the message: "Fight Pollution: The World You Save May Be Your Own," and would be set up and removed by a crew from the requester's organization. If the $\mathrm{S}$ agreed, she was told that she would receive a call to let her know when the sign would be delivered, and was thanked for her cooperation. If she refused, she was thanked for her courtesy in listening to the request. 


\section{One-Contact Group}

On the same days that they paid their second visits to $S$ s in the two-contact groups. the $F$ also contacted individuals who had not been seen before and asked their permission to set up the billboard on their lawns. In this condition. a precaution against the Ss' obtaining knowledge of the experimental procedures from their neighbors. each investigator moved to a new but nearby street before collecting data for this group.

\section{RESULTS}

Fully $95 \%$ of the Ss contacted agreed to the initial requests made by the Es. Moreover, this figure was identical in the small and moderate first-request conditions, and did not vary with the sex of the requester. Thus, as anticipated on the basis of pilot data, there were no differences between the various groups with respect to such compliance.

Turning to compliance with the large request, it was found that among Ss visited by a female $\mathrm{E}$, there were no significant differences between the three experimental conditions (approximately 20\%-25\% agreed in each case). Among individuals visited by a male $\mathrm{E}$, however, it was found that a significantly larger percentage of Ss in the small-first-request condition agreed to the placement of the sign on their lawns than did those in the one-contact control group (50\% vs $20 \%$, $\mathrm{p}<.05$, by appropriate chi square). Thus, it appeared that the "foot-in-the-door" effect occurred among Ss in this group. In contrast, the percentage of individuals complying in the moderate-first-request group was not significantly larger than that in the one-contact control condition $(p>10)$. It appeared, therefore, that an initial request was successful in increasing later compliance only when it was of very small magnitude.

\section{DISCUSSION}

The hypothesis that $\mathrm{Ss}$ in the moderate-first-request condition would be more likely to comply with a second, large request than those in the small-first-request condition was not confirmed. Instead, it was found that only $\mathrm{Ss}$ in the small-first-request group demonstrated a higher level of compliance than those in the one-contact control condition. One possible explanation for this unexpected pattern of findings is suggested by the fact that several of the individuals in the moderate-first-request condition who refused to perform the large request explained their refusal by stating that they had already "done enough" for the E's organization, and that it was unfair of him or her to ask them to perform still another task. (No Ss in the small-initial-request group were heard to make such comments.) These statements by Ss suggest very strongly that they may have experienced feelings of psychological reactance (sec Brehm, 1966), which tended to reduce their willingness to comply with the large, second request. If this was indeed the case. then the "foot-in-the-door" technique may prove to be effective in increasing later compliance only under conditions where the magnitude of the initial request is small enough to avoid the induction of such feelings among Ss. Further experiments designed to examine this interesting possibility are currently planned.

The finding that the "foot-in-the-door" effect was obtained only with Ss who were approached by male Es may be attributable to the fact that these individuals were viewed as somewhat more competant and knowledgeable than the female investigators with regard to the particular issue involved (i.e., pollution of the environment). A considerable body of research (e.g.. Baron. 1970; Hovland \& Weiss, 1951) suggests that the success of social influence attempts may be strongly affected by the apparent competence or expertise of the influencing agent, and it seems reasonable to suggest that this factor may also play a role in determining the magnitude of the "foot-in-the-door" effect. This possibility may be readily examined in future experiments by varying the apparent competence of the requester in a systematic manner.

Regardless of the mechanisms which are ultimately shown to be responsible for the present pattern of findings, however, it appears that the "foot-in-the-door" phenomenon may occur only under more specific" and restricted conditions than was originally suggested by the findings of the Freedman \& Fraser (1966) experiment. Thus, it may prove to be somewhat less effective in producing compliance in the absence of overt social pressure than has previously been believed.

\section{REFERENCES}

Baron, R. A. Attraction toward the model and model's competence as determinants of adult imitative behavior. Journal of Personality \& Social Psychology, 1970, 14, 345-351.

Brehm, J. W. A theory of psychological reactance. New York: Academic Press, 1966.

Freedman, J. L., \& Fraser, S. C. Compliance without pressure: The foot-in-the-door technique. Journal of Personality \& Social Psychology, 1966, 4, 195-202.

Helson, H. Adaptation-level as a basis for a quantitative theory of frames of reference. Psychological Review, 1948, 55, 297-313.

Hovland, C. I., \& Weiss, W. The influence of source credibility on communication effectiveness. Public Opinion Quarterly, 1951, 15, 635-650.

(Received for publication May 4, 1973.) 